\title{
Formation and characterization of varied size germanium nanocrystals by electron microscopy, Raman spectroscopy, and photoluminescence
}

\author{
Ou, Haiyan; Ou, Yiyu; Liu, Chuan; Berg, Rolf W.; Rottwitt, Karsten
}

Published in:

Optical Materials Express

Publication date:

2011

Document Version

Publisher's PDF, also known as Version of record

Link back to DTU Orbit

Citation (APA):

Ou, H., Ou, Y., Liu, C., Berg, R. W., \& Rottwitt, K. (2011). Formation and characterization of varied size germanium nanocrystals by electron microscopy, Raman spectroscopy, and photoluminescence. Optical Materials Express, 1(4), 643-651.

\section{General rights}

Copyright and moral rights for the publications made accessible in the public portal are retained by the authors and/or other copyright owners and it is a condition of accessing publications that users recognise and abide by the legal requirements associated with these rights.

- Users may download and print one copy of any publication from the public portal for the purpose of private study or research.

- You may not further distribute the material or use it for any profit-making activity or commercial gain

- You may freely distribute the URL identifying the publication in the public portal 


\title{
Formation and characterization of varied size germanium nanocrystals by electron microscopy, Raman spectroscopy, and photoluminescence
}

\author{
Haiyan Ou, ${ }^{1, *}$ Yiyu Ou, ${ }^{1}$ Chuan Liu, ${ }^{2}$ Rolf W. Berg, ${ }^{2}$ and Karsten Rottwitt ${ }^{1}$ \\ ${ }^{I}$ Department of Photonics Engineering, Technical University of Denmark, DK-2800, Kgs. Lyngby, Denmark \\ ${ }^{2}$ Department of Chemistry, Technical University of Denmark, DK-2800, Kgs. Lyngby, Denmark \\ *haou@fotonik.dtu.dk
}

\begin{abstract}
Germanium nanocrystals are being extensively examined. Their unique optical properties (brought about by the quantum confinement effect) could potentially be applied in wide areas of nonlinear optics, light emission and solid state memory etc. In this paper, Ge nanocrystals embedded in $\mathrm{SiO}_{2}$ matrix were formed by complementary metal-oxidesemiconductor compatible technology, e.g. plasma enhanced chemical vapour deposition and annealing. Different sizes of the Ge nanocrystals were prepared and analyzed by transmission electron microscopy with respect to their size, distribution and crystallization. The samples of different size $\mathrm{Ge}$ nanocrystals embedded in the $\mathrm{SiO}_{2}$ matrix were characterized by Raman spectroscopy and photoluminescence. Interplayed size and strain effect of Ge nanocystals was demonstrated by Raman spectroscopy after excluding the thermal effect with proper excitation laser power. It was clarified that two strong emission peaks at $3.19 \mathrm{eV}$ and 4.40 $\mathrm{eV}$ are from the interface between $\mathrm{Ge}$ nanocrystals and $\mathrm{SiO}_{2}$ matrix.
\end{abstract}

(C)2011 Optical Society of America

OCIS codes: (160.4236) Nanomaterials; (160.6000) Semiconductor materials; (300.0300) Spectroscopy; (250.5230) Photoluminescence.

\footnotetext{
References and links

1. A. Rodríguez, M. I. Ortiz, J. Sangrador, T. Rodriguez, M. Avella, A. C. Prieto, A. Torres, J. Jimenez, A. Kling, and C. Ballesteros, "Comparative study of the luminescence of structures with Ge nanocrystals formed by dry and wet oxidation of SiGe films," Nanotechnology 18(6), 065702 (2007).

2. T. V. Torchynska, J. Aguilar-Hernandex, L. Schacht Hernandez, G. Polupan, Y. Goldstein, A. Many, J. Jedrzejewski, and A. Kolobov, "Mechanism of photoluminescence of silicon oxide films enriched by Si and Ge," Microelectron. Eng. 66(1-4), 83-90 (2003).

3. W. K. Choi, Y. W. Ho, S. P. Ng, and V. Ng, "Microstructural and photoluminescence studies of germanium nanocrystals in amorphous silicon oxide films," J. Appl. Phys. 89(4), 2168-2172 (2001).

4. Y. Maeda, "Visible photoluminescence from nanocrystallite Ge embedded in a glassy $\mathrm{SiO}_{2}$ matrix: Evidence in support of the quantum-confinement mechanism," Phys. Rev. B Condens. Matter 51(3), 1658-1670 (1995).

5. A. K. Dutta, "Visible photoluminescence from Ge nanocrystal embedded into a $\mathrm{SiO}_{2}$ matrix fabricated by atmospheric pressure chemical vapor deposition," Appl. Phys. Lett. 68(9), 1189-1191 (1996).

6. H. Yang, X. Yao, S. Xie, X. Wang, S. Liu, Y. Fang, X. Gu, and F. Wang, "Structure and photoluminescence of Ge nanoparticles embedded in $\mathrm{SiO}_{2}$ gel glasses fabricated at different temperatures," Opt. Mater. 27(4), 725-730 (2005).

7. C. L. Heng, Y. J. Liu, A. T. S. Wee, and T. G. Finstad, "The formation of Ge nanocrystals in a metal-insulatorsemiconductor structure and its memory effect," J. Cryst. Growth 262(1-4), 95-104 (2004).

8. M. Kanoun, C. Busseret, A. Poncet, A. Souifi, T. Baron, and E. Gautier, "Electronic properties of Ge nanocrystals for non volatile memory applications," Solid-State Electron. 50(7-8), 1310-1314 (2006).

9. H. P. Li, C. H. Kam, Y. L. Lam, Y. X. Jie, W. Ji, A. T. S. Wee, and C. H. A. Huan, "Nonlinear optical response of Ge nanocrystals in silica matrix with excitation of femtosecond pulses," Appl. Phys. B 72, 611-615 (2001).

10. Y. X. Jie, Y. N. Xiong, A. T. S. Wee, C. H. A. Huan, and W. Ji, "Dynamics of optical nonlinearity of Ge nanocrystals in a silica matrix," Appl. Phys. Lett. 77(24), 3926-3928 (2000).

11. A. Dowd, R. G. Elliman, M. Samoc, and B. Luther-Davies, "Nonlinear optical response of Ge nanocrystals in a silica matrix," Appl. Phys. Lett. 74(2), 239-241 (1999).

12. L. P. Yue and Y.Z. He, "A study of the nonlinear absorption of Ge nanocrystallites embedded in $\mathrm{SiO}_{2}$ thin films by the z-scan technique," J. Mater. Sci. Lett. 15(3), 263-265 (1996).
} 
13. X. Sun, J. Liu, L. C. Kimerling, and J. Michel, "Toward a germanium laser for integrated silicon photonics," IEEE J. Sel. Top. Quantum Electron. 16(1), 124-131 (2010).

14. J. Liu, X. Sun, D. Pan, X. Wang, L. C. Kimerling, T. L. Koch, and J. Michel, "Tensile-strained, n-type Ge as a gain medium for monolithic laser integration on Si," Opt. Express 15(18), 11272-11277 (2007).

15. Y. Kanemitsu, H. Uto, Y. Masumoto, and Y. Maeda, "On the origin of visible photoluminescence in nanometersize Ge crystallites,” Appl. Phys. Lett. 61(18), 2187-2189 (1992).

16. E. B. Kaganovich, D. V. Korbutyak, Yu. V. Kryuchenko, I. M. Kupchak, E. G. Manoilov, and A. V. Sachenko, "Exciton states and photoluminescence in Ge quantum dots," Nanotechnology 18(29), 295401 (2007).

17. S. Dun, T. Lu, Q. Hu, Y. Hu, C. You, S. Zhang, B. Tang, J. Dai, and N. Huang, "Photoluminescence study of Ge nanocrystals irradiated by reactor neutron flux," Nucl. Instrum. Methods Phys. Res. B 264(2), 272-276 (2007).

18. S. T. Chang and S. H. Liao, "Light emission and photoluminescence from high-k dielectrics containing Ge nanocrystals," J. Vac. Sci. Technol. B 27(1), 535-537 (2009).

19. L. Nataraj, F. Xu, and S. G. Cloutier, "Direct-bandgap luminescence at room-temperature from highly-strained Germanium nanocrystals,” Opt. Express 18(7), 7085-7091 (2010).

20. Y. Kanemitsu, K. Masuda, M. Yamamoto, K. Kajiyama, and T. Kushida, "Near-infrared photoluminescence from Ge nanocrystals in $\mathrm{SiO}_{2}$ matrices," J. Lumin. 87-89, 457-459 (2000).

21. S. Takeoka, M. Fujii, S. Hayashi, and K. Yamamoto, "Size-dependent near-infrared photoluminescence from Ge nanocrystals embedded in $\mathrm{SiO}_{2}$ matrices," Phys. Rev. B 58(12), 7921-7925 (1998).

22. J. S. Jensen, T. P. L. Pedersen, R. Pereira, J. Chevallier, J. L. Hansen, B. B. Nielsen, and A. N. Larsen, “Ge nanocrystals in magnetron sputtered $\mathrm{SiO}_{2}$," Appl. Phys., A Mater. Sci. Process. 83(1), 41-48 (2006).

23. J. M. J. Lopes, F. C. Zawislak, M. Behar, P. F. P. Fichtner, L. Rebohle, and W. Skorupa, "Cluster coarsening and luminescence emission intensity of Ge nanoclusters in $\mathrm{SiO}_{2}$ layers," J. Appl. Phys. 94(9), 6059-6064 (2003).

24. S. Dun, T. Lu, Q. Hu, Y. Hu, C. You, S. Zhang, B. Tang, J. Dai, and N. Huang, "Photoluminescence study of Ge nanocrystals irradiated by reactor neutron flux," Nucl. Instrum. Methods Phys. Res. B 264(2), 272-276 (2007).

25. A. G. Rolo, A. Chahboun, O. Conde, M. I. Vasilevskiy, and M. J. M. Gomes, "Annealing effect on the photoluminescence of Ge-doped silica films," Physica E 40(3), 674-679 (2008).

26. P. K. Giri, S. Bhattacharyya, K. Das, S. K. Roy, R. Kesavamoorthy, B. K. Panigrahi, and K. G. M. Nair, “A comparative study of the vibrational and luminescence properties of embedded Ge nanocrystals prepared by ion implantation and sputter deposition methods: role of strain and defects," Semicond. Sci. Technol. 22(12), 13321338 (2007).

27. Y. Wang, Y. Yang, Y. Guo, J. Yue, and R. Gan, "Raman scattering and room-temperature visible photoluminescence from Ge nanocrystals embedded in $\mathrm{SiO}_{2}$ thin films," Mater. Lett. 29(1-3), 159-164 (1996).

28. H. Ou, T. P. Rørdam, K. Rottwitt, F. Grumsen, A. Horsewell, R. W. Berg, and P. Shi, "Ge nanoclusters in PECVD-deposited glass caused only by heat treatment,” Appl. Phys. B 91(1), 177-181 (2008).

29. H. Ou, T. P. Rørdam, K. Rottwitt, F. Grumsen, A. Horsewell, and R. W. Berg, "Ge nanoclusters in PECVDdeposited glass after heat treatment and electron-beam irradiation," Appl. Phys. B 87(2), 327-331 (2007).

30. H. Ou, T. P. Rørdam, K. Rottwitt, F. Grumsen, and A. Horsewell, "Ge-nanoclusters embedded in Ge-doped sililca-on-silicon waveguides," Electron. Lett. 42(9), 532-534 (2006).

31. R. W. Berg and T. Nørbygaard, "Wavenumber calibration of CCD detector Raman spectrometers controlled by a sinus arm drive," Appl. Spectrosc. Rev. 41(2), 165-183 (2006).

32. L. Skuja, "Isoelectronic series of twofold corrdinated $\mathrm{Si}, \mathrm{Ge}$, and $\mathrm{Sn}$ atoms in glassy $\mathrm{SiO}_{2}$ : a luminescence study,” J. Non-Cryst. Solids 149(1-2), 77-95 (1992).

33. P. K. Giri, S. Bhattacharyya, S. Kumari, K. Das, S. K. Ray, B. K. Panigrahi, and K. G. M. Nair, "Ultraviolet and blue photoluminescence from sputter deposited Ge nanocrystals embedded in $\mathrm{SiO}_{2}$ matrix," J. Appl. Phys. 103(10), 103534 (2008).

\section{Introduction}

Zero-dimensional germanium (Ge) nanocrystals (ncs), also called nanoclusters or quantum dots, have undergone intensive theoretical and experimental research due to their potential applications as light emitters [1-6], non-volatile optical memories [7,8] and their enhanced third-order optical nonlinear effects [9-12], etc.

Among the applications, the high-efficiency Ge light emitter application is receiving tremendous attention because $\mathrm{Ge}$ has a direct bandgap $(0.80 \mathrm{eV})$ only slightly larger (by 0.136 $\mathrm{eV})$ than its indirect bandgap $(0.664 \mathrm{eV})$, putting $\mathrm{Ge}$ emission at high efficiency within the third optical communication window $(\sim 1520-1620 \mathrm{~nm})$. It has been reported that bulk Ge could emit with $10 \%$ efficiency at its direct bandgap wavelength of $1550 \mathrm{~nm}$ by optimized doping and strain implementation $[13,14]$. By tuning the strain, the emission wavelength could be tuned between $1238 \mathrm{~nm}$ and $2476 \mathrm{~nm}$ [13]. The emission wavelengths can also be tuned between $1548 \mathrm{~nm}$ and $1905 \mathrm{~nm}$ by changing the doping levels [13]. Ge ncs were reported to emit between $563 \mathrm{~nm}$ and $1548 \mathrm{~nm}$ explained by the quantum confinement effect (QCE) [15-21]. In addition, many results have been published covering the entire visible and ultraviolet (UV) ranges related to various defects or interface of Ge ncs with matrix $[18,22$ 27].

\#148333 - \$15.00 USD Received 27 May 2011; revised 11 Jul 2011; accepted 11 Jul 2011; published 18 Jul 2011

(C) 2011 OSA

1 August 2011 / Vol. 1, No. 4 / OPTICAL MATERIALS EXPRESS 644 
Based on these published results, it is very promising to achieve a highly efficient Ge ncs light emitter within the third optical communication window with combined effects of QCE, strain and doping. To reach such a goal, size and strain effects play very important roles, and therefore should be studied carefully.

In this paper, $\mathrm{Ge}$ ncs embedded in $\mathrm{SiO}_{2}$ matrix were formed successfully by a complementary metal-oxide-semiconductor (CMOS) compatible technology, e.g. plasma enhanced chemical vapour deposition (PECVD) and annealing, which is very unique. One big potential application of these Ge ncs is to make a monolithically integrated light source for silicon ( $\mathrm{Si}$ ) photonics, which is a still missing crucial building block. The successful demonstration of strong light emission from Ge ncs made by CMOS compatible processing will be implemented easily to a wide range of Si based optoelectronic devices. Different sizes of the Ge ncs were obtained by tuning the ratio of $\mathrm{Ge} / \mathrm{Si}$ in a SiGe alloy layer. The Ge ncs were analyzed by transmission electron microscopy (TEM) in terms of their size, distribution and crystallization. The samples of different size Ge ncs embedded in $\mathrm{SiO}_{2}$ matrix were characterized by Raman spectroscopy. It is clearly shown that the Raman peak position and its full width at half height (FWHM) are dependent both on the sizes and on the strain of the Ge ncs. How to separate the effects of size and strain will be explored further in future. Moreover, photoluminescence of the samples from 250 to $500 \mathrm{~nm}$ were measured and compared to a $\mathrm{SiO}_{2}$ sample without Ge ncs at an excitation wavelength of $244 \mathrm{~nm}$. It is clarified that two strong peaks at $3.19 \mathrm{eV}$ and $4.40 \mathrm{eV}$ are not from Ge ncs, but from the interface of Ge ncs with the $\mathrm{SiO}_{2}$ matrix.

\section{Sample Preparation}

Five samples (Ge0, Ge0.25, Ge0.5, Ge1_A and Ge1_B) have been prepared by using PECVD deposition and annealing. We started with 4 inch (100) oriented Si substrates. First, one layer of $\mathrm{Ge}$ doped $\mathrm{SiO}_{2}$ was deposited. Then a $\mathrm{SiGe}$ alloy layer was deposited on top of the $\mathrm{SiO}_{2}$ layer. The ratios of the Si to Ge were tuned from 1:1 to 1:0.5 and 1:0.25 for samples Ge1_A, $\mathrm{Ge} 0.5$ and $\mathrm{Ge} 0.25$, respectively. Thirdly, one identical layer of $\mathrm{Ge}$ doped $\mathrm{SiO}_{2}$ was deposited on the $\mathrm{SiGe}$ alloy layer. By repeating alternate layers of $\mathrm{SiGe}$ alloy layer and $\mathrm{Ge}$ doped $\mathrm{SiO}_{2}$ layer, a multilayered structure was readily made by means of PECVD deposition. A 5-layer structure is shown schematically in Fig. 1 . The samples were annealed at $1100{ }^{\circ} \mathrm{C}$ in $\mathrm{N}_{2}$ for 4 hours after deposition. More details of the fabrication parameters are found in [28].

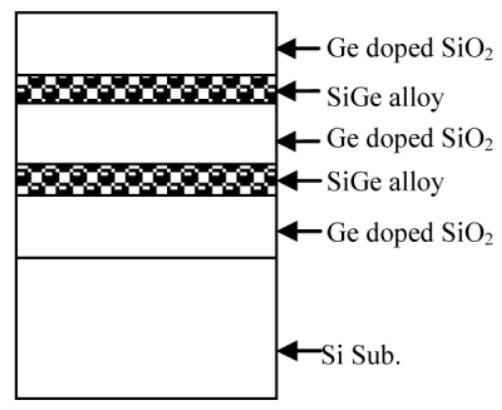

Fig. 1. A schematic drawing of the multilayer structure.

The 5 different samples prepared (Ge0, Ge0.25, Ge0.5, Ge1_A and Ge1_B) are summarized in Table 1.

Table 1. Summary of Samples Prepared and Their Structures

\begin{tabular}{l|l|l}
\hline \multicolumn{1}{c|}{ Sample ID } & \multicolumn{1}{|c}{ Si/Ge ratio } & \multicolumn{1}{c}{ Layer structure } \\
\hline $\mathrm{Ge} 0$ & & $\mathrm{Si} \mathrm{Sub.}+\mathrm{SiO}_{2}$ \\
$\mathrm{Ge} 0.25$ & $1: 0.25$ & $\mathrm{Si} \mathrm{Sub}+\mathrm{SiO}_{2}+\mathrm{SiGe}+\mathrm{SiO}_{2}$ \\
$\mathrm{Ge} 0.5$ & $1: 0.5$ & $\mathrm{Si} \mathrm{Sub}+\mathrm{SiO}_{2}+\mathrm{SiGe}+\mathrm{SiO}_{2}$ \\
$\mathrm{Ge} 1 \_\mathrm{A}$ & $1: 1$ & $\mathrm{Si} \mathrm{Sub}+\mathrm{SiO}_{2}+\mathrm{SiGe}+\mathrm{SiO}_{2}$ \\
$\mathrm{Ge} 1 \_B$ & $1: 1$ & $\mathrm{Si} \mathrm{sub}+\mathrm{SiO}_{2}+\mathrm{SiGe}+\mathrm{SiO}_{2}+\mathrm{SiGe}+\mathrm{SiO}_{2}$ \\
\hline
\end{tabular}

\#148333 - \$15.00 USD Received 27 May 2011; revised 11 Jul 2011; accepted 11 Jul 2011; published 18 Jul 2011

(C) 2011 OSA 1 August 2011 / Vol. 1, No. 4 / OPTICAL MATERIALS EXPRESS 645 
From Table 1, one sees that sample Ge0 didn't contain any Ge ncs because it didn't have a SiGe alloy layer. From our previous results in [29], we know that heat treatment doesn't trigger the formation of Ge ncs, even in very heavily Ge doped $\mathrm{SiO}_{2}$. Samples Ge0.25, Ge0.5 and Ge1_A had different Si/Ge ratios in the SiGe alloy layer for comparison. All of them had 3-layer structures. Sample Ge1_B had a 5-layer structure, made in order to increase the Ge nanocrystal concentration.

\section{TEM and SEM Analysis}

The 3 samples (Ge0.25, Ge0.5 and Ge1_A) were firstly examined by TEM (by use of a FEI Tecnai G2 apparatus) with focus on size, distribution and crystalline state of the Ge ncs. The scanning electron microscopy (SEM) image for sample Ge1_B was taken by use of a Leo 1550 instrument. A detailed investigation of one sample with 1:1 Si/Ge ratio is found in [28].

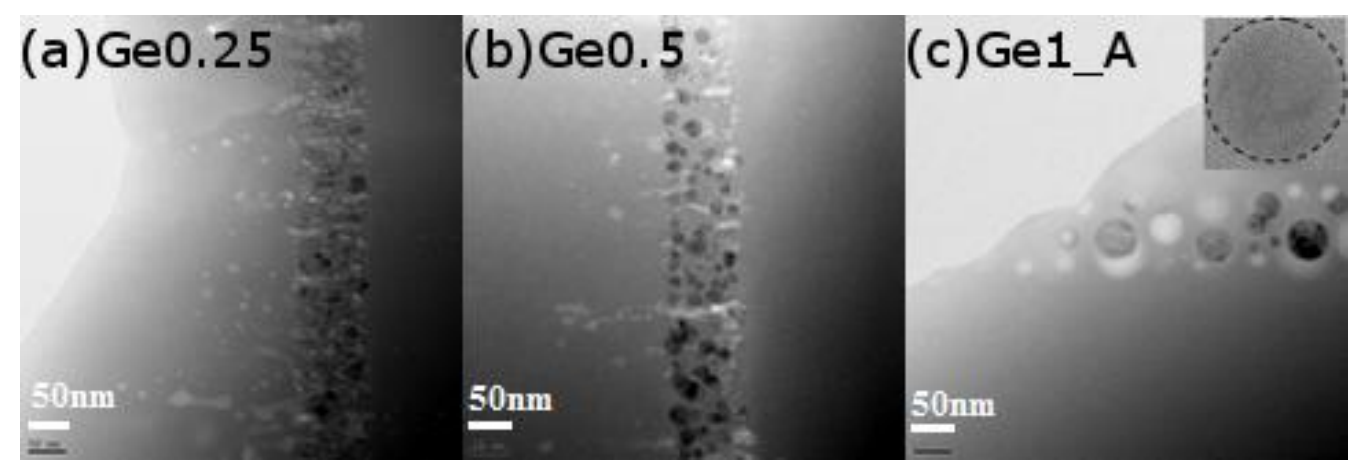

Fig. 2. TEM cross-sectional images of samples Ge0.25(a), Ge0.5(b) and Ge1_A(c).

TEM cross-sectional images of samples Ge0.25, Ge0.5 and Ge1_A are shown in Figs. 2(a), 2(b) and 2(c) respectively. The inset in Fig. 2(c) is a high resolution TEM image showing crystal planes of one round cluster, which gives the evidence that the clusters are crystalline. Then the spot size of the TEM was focused to one cluster and the EDX analysis showed two elements: 7.65 atomic\% $\mathrm{Si}$ and 92.35 atomic\% Ge. From HRTEM and EDX analysis, it could be concluded that the black dots appearing in the TEM images were crystalline Ge nanoclusters. Figure 2 shows the presence of both Ge ncs (black dots) and voids (white dots) in the as-deposited $\mathrm{SiGe}$ alloy layer, which was sandwiched by the $\mathrm{SiO}_{2}$ layer. For sample Ge0.25, the diameter of the Ge ncs was within $25 \mathrm{~nm}$. Some of the voids even seemed to have diffused to the top surface of the wafer. For sample Ge0.5, both Ge ncs and voids presented themselves with sizes larger than that for sample Ge0.25. The Ge ncs had diameters up to $30 \mathrm{~nm}$. For sample Ge1, the Ge ncs had variable diameters up to $80 \mathrm{~nm}$. The Ge ncs in all three samples were crystalline (checked by HR image). Since high energy electron irradiation during the TEM examination may have initiated the formation of or changed the Ge ncs [30], the TEM images in Fig. 2 were taken from fresh surface areas. It can be concluded from Fig. 2 that the size of Ge ncs increases as the ratio of $\mathrm{Ge} / \mathrm{Si}$ increases. 


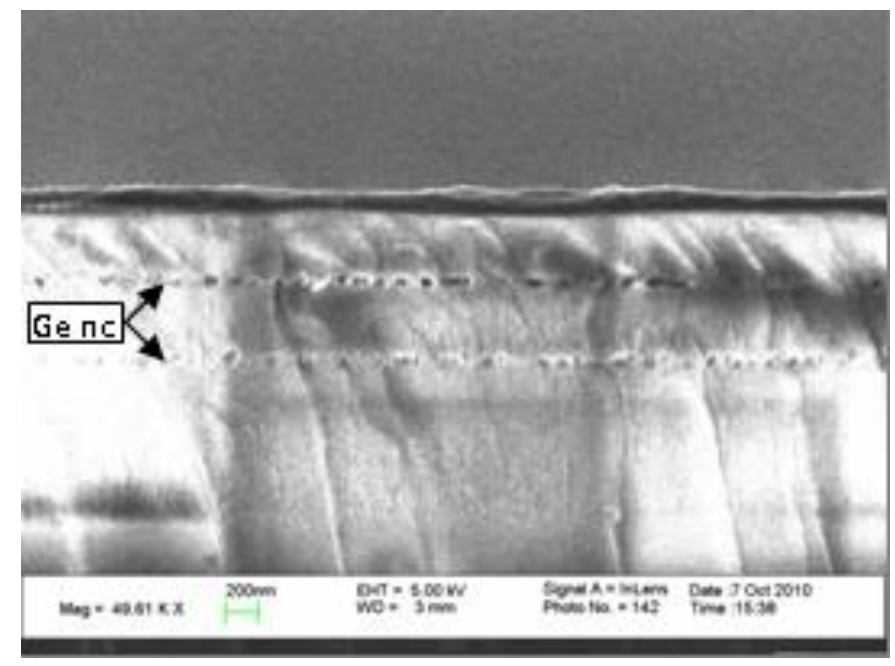

Fig. 3. SEM cross-sectional image of sample Ge1_B.

A cross-sectional SEM image of sample Ge1_B is shown in Fig. 3. Sample Ge1_B was deposited as a 5-layer structure. Two layers of Ge ncs buried in the core of the waveguide are shown in the image. The maximum diameter of the Ge ncs in sample Ge1_B was 120 nm.

\section{Raman Spectroscopy}

The Raman spectra were measured at room temperature by using a RENISHAW inVia Raman Microscope. It is configured as a backscattering collection system with a microscope sampling stage. Both the excitation and the light collection are coupled (in and out the microscope) by means of high-pass interference filters. The system was calibrated by use of cyclohexane and the measurement resolution was $\sim 1.3 \mathrm{~cm}^{-1}$. Details of the calibration can be found in [31]. The excitation laser had a wavelength of $488 \mathrm{~nm}$. An X20 objective lens was used (corresponding to a beam spot size of $1.5 \mu \mathrm{m}$ ). The power was measured by a power meter (Thorlabs PM30-120V) directly under the objective lens.

According to Nataraj et al. [19], the Ge ncs Raman peak shifts its position linearly with the excitation power density due to the thermal effect. Special attention should be paid to the nanocrystals because their Raman signal usually is weak. In order to get a measurable signal, a high excitation power or long counting times or both have to be applied. Care is particularly important in the present case, because our Ge ncs are embedded in a $\mathrm{SiO}_{2}$ matrix, which has a quite poor thermal dissipation. In order to exclude influences from any thermal effect, we measured the Raman spectra of sample Ge1_B with varied excitation power levels ranging from $1 \mathrm{~mW}$ to $21 \mathrm{~mW}$. The results are shown in Fig. 4(a). The changes of the Ge Raman peak shifts and the FWHM values with different excitation powers from Fig. 4(a) are shown in Fig. 4(b). 


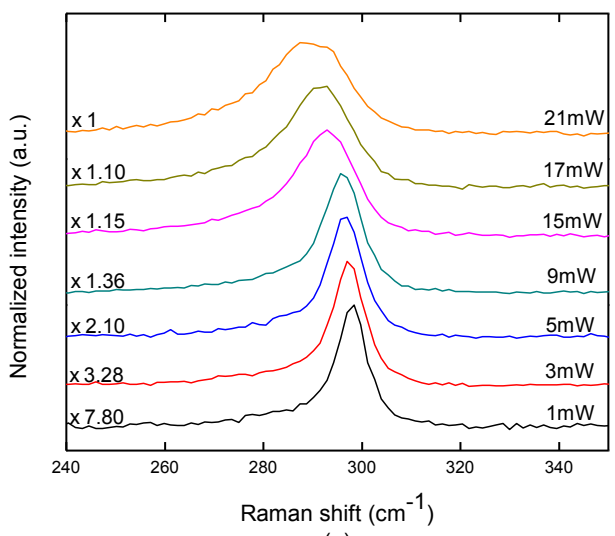

(a)

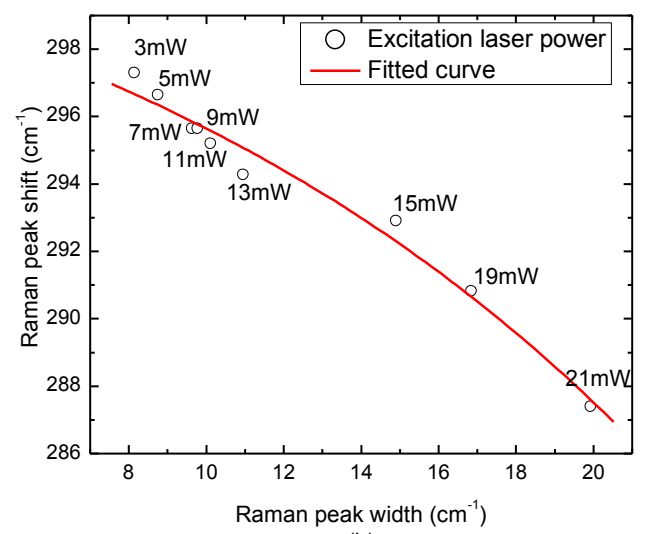

(b)

Fig. 4. (a) Raman spectra for sample Ge1_B with varied excitation powers from $1 \mathrm{~mW}$ to $21 \mathrm{~mW}$, (b) Raman peak shift and FWHM with relation to the excitation powers.

Figure 4(a) shows that as excitation power increases, the Ge Raman peak has a downshift and the line width increases. At low excitation powers $(<11 \mathrm{~mW})$, there is no obvious linear relationship between the shift and the excitation power. However, at high excitation powers, the heat effect starts to play a dominant role, and a linear relationship is emerging. Therefore, the following Raman spectra were recorded by using an excitation power of $5 \mathrm{~mW}$, to reveal the properties, for example the size-dependent effect and the strain effect, unbiased by any heat from the Raman excitation light.

The Raman spectra of samples Ge0.25, Ge0.5, Ge1_A and Ge1_B together with a singlecrystalline Ge wafer (as a reference), are shown in Fig. 5, all obtained with an excitation power of $5 \mathrm{~mW}$ and a $10 \mathrm{~s}$ integration time. The reference Ge wafer showed a strong Raman peak at $298.6 \mathrm{~cm}^{-1}$. All the other samples showed two distinct peaks: one at around $300 \mathrm{~cm}^{-1}$, corresponding to the Ge ncs and one at around $520 \mathrm{~cm}^{-1}$, corresponding to the Si substrate. The appearance of the peak at $300 \mathrm{~cm}^{-1}$ further confirms the formation of Ge ncs, adding to the results from the TEM examination. An enlarged zoom of the Ge peak is inserted in Fig. 5. The detailed information with respect to the Ge Raman peak shifts and the FWHM data are listed in Table 2. Compared to the reference Ge wafer, the Ge peaks for the 4 samples all appeared shifted. Samples Ge0.25, Ge0.5 and Ge1_A all had upshifts and sample Ge1_B had a downshift. 


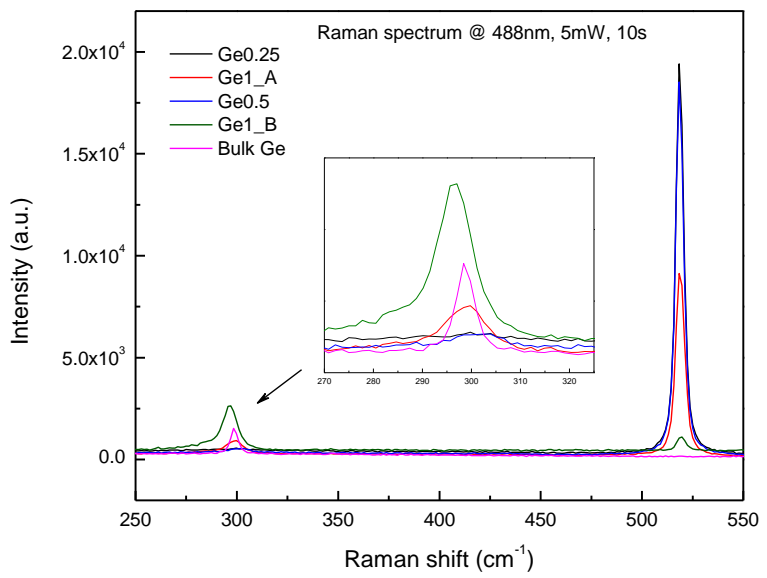

Fig. 5. Raman spectra of samples Ge0.25, Ge0.5, Ge1_A, Ge1_B and the reference Ge wafer. The inset is a zoom-in on the Ge peak.

Table 2. Detailed Information on the Ge Nanocrystal Size, the Raman Peak Shifts and the FWHMs for Samples Ge0.25, Ge0.5, Ge1_A, Ge1_B, Compared to the Ge Wafer Reference

\begin{tabular}{llll}
\hline Sample & \multicolumn{1}{c}{ Max. nanocrystal size $(\mathrm{nm})$} & \multicolumn{1}{c}{ Raman shift $\left(\mathrm{cm}^{-1}\right)$} & FWHM $\left(\mathrm{cm}^{-1}\right)$ \\
\hline Bulk Ge & Infinity & 0 & 4.46 \\
Ge0.25 & 25 & 1.375 & $8.3^{*}$ \\
Ge0.5 & 30 & 4.125 & $14.8^{*}$ \\
Ge1_A & 80 & 1.375 & 10.12 \\
Ge1_B & 120 & -1.376 & 8.77
\end{tabular}

*The signals from samples Ge0.25 and Ge0.5 were rather weak, and the readings of their FWHM values had relatively large degrees of uncertainty.

Size effects, thermal effects and strain are possible reasons for the peak shifts. Results shown in Fig. 5 may be taken as a proof to exclude any thermal effect. So the shifts are only dependent on the two other effects, i.e. size and strain effects. From Table 2, no simple relationship between the Raman shift and the Ge nanocrystal size seems to be obvious, which implies that the size effect and strain probably are interplayed and difficult to distinguish in this case. A template assisted method to form uniform Ge ncs by PECVD and annealing is being developed at the moment, and we hope in the future to be able to control the size of the Ge ncs and in this way to separate the effects of size and strain.

\section{Photoluminescence}

Photoluminescence of samples $\mathrm{Ge} 0, \mathrm{Ge} 0.25, \mathrm{Ge} 0.5$, Ge1_A and $\mathrm{Ge} 1 \_\mathrm{B}$ was measured by using the same system as for the Raman spectroscopy measurements, but with an excitation wavelength of $244 \mathrm{~nm}$, an objective lens of X15 magnification (corresponding to a beam spot size of $0.93 \mu \mathrm{m}$ ), and a detection range from $250 \mathrm{~nm}$ to $500 \mathrm{~nm}$. The results are shown in Fig. 6.

For sample Ge0, there is no photoluminescence at all, which implies that the emission is not from defects of the $\mathrm{SiO}_{2}$ matrix. For the rest of the samples $(\mathrm{Ge} 0.25, \mathrm{Ge} 0.5, \mathrm{Ge} 1 \mathrm{~A}$ and Ge1_B), all of them had two strong peaks at $3.19 \mathrm{eV}$ and $4.40 \mathrm{eV}$. The fixed emission peaks 
from the different samples with varied sizes indicate that the emission did not come directly from the Ge ncs. Instead, they were mostly related to the interface between Ge ncs and the $\mathrm{SiO}_{2}$ matrix. Those two peaks at $3.19 \mathrm{eV}$ and $4.40 \mathrm{eV}$ have been observed by Skuja [32], Lopes [23], and Rodriguez [1], who attributed them to defects, formed by electrically neutral twofold-coordinated $\mathrm{Ge}$ atoms. These molecule-like luminescence centers have three-level energy emission in which blue-violet emission $(3.19 \mathrm{eV})$ and $\mathrm{UV}$ emission $(4.40 \mathrm{eV})$ are due to a triplet-to-singlet transmission and a singlet-to-singlet transition, respectively [23]. Giri [33] has also observed the peak at $3.19 \mathrm{eV}$ from sputter deposited Ge nanocrystals embedded in $\mathrm{SiO}_{2}$ matrix and also assigned this peak to the defective $\mathrm{Ge} n \mathrm{~ns} / \mathrm{SiO}_{2}$ interface. In samples (Ge0.25, Ge0.5, Ge1_A and Ge1_B), there are two sources for these oxygen deficient $\mathrm{Ge}$ centers: one is from Ge doped $\mathrm{SiO}_{2}$ film and the other one is from the interface between $\mathrm{Ge}$ ncs and the surrounding $\mathrm{SiO}_{2}$ matrix. From Fig. 6, the intensity ratio of the peak at $4.40 \mathrm{eV}$ to that at $3.19 \mathrm{eV}$ increases from Ge1_A over Ge0.5 to Ge0.25, which is also very interesting information needed to be clarified further. The ripples in each spectrum are caused by the Fabry-Perot cavity between the two reflective interfaces (air/SiO 2 and $\mathrm{SiO}_{2} / \mathrm{Si}$ substrate). The calculated cavity length $(2.5 \mu \mathrm{m}$ for $\mathrm{Ge} 0.25)$ according to the wave period from the spectrum agrees well with the TEM characterization results of the sample (the total thickness of the 3layer was $2.3 \mu \mathrm{m}$ ). A new setup is being built to measure the luminescence in the IR range.

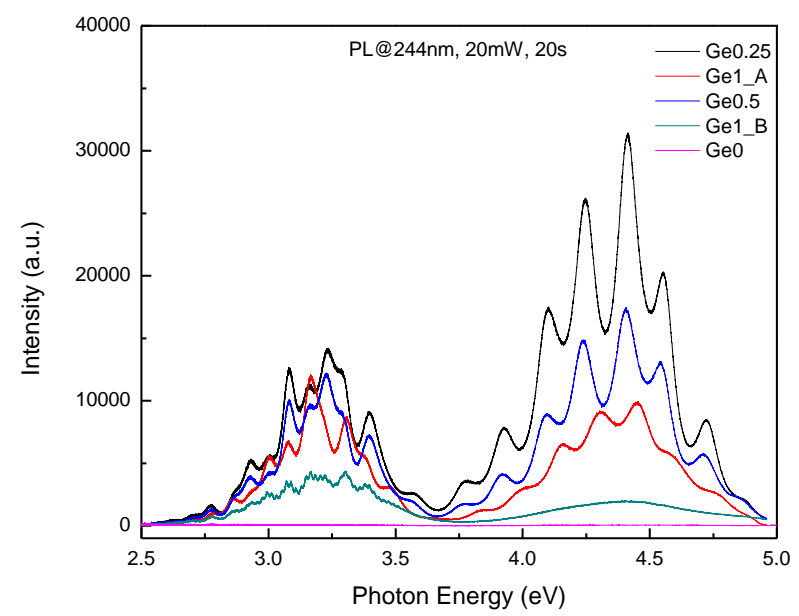

Fig. 6. Photoluminescence of samples Ge0, Ge0.25, Ge0.5, Ge1_A and Ge1_B.

\section{Conclusion}

Ge nanocrystals of different sizes have been successfully formed by tuning the Si/Ge ratio in the as-deposited SiGe alloy layer. The size and distribution of the samples have been investigated by TEM. The existence of the Ge ncs in the $\mathrm{SiO}_{2}$ matrix has been confirmed by both TEM analysis and Raman spectroscopy. Suitable low excitation power levels for Raman spectroscopy to exclude any thermal effect were selected by measuring the Raman spectra within a large excitation power range from $1 \mathrm{~mW}$ to $21 \mathrm{~mW}$. From the Raman measurements, the observed peak shifts (after excluding the thermal effect) were assigned to interplaying phenomena such as size and strain effects. It was clarified through photoluminescence measurements that the two strong emission peaks at $3.19 \mathrm{eV}$ and $4.40 \mathrm{eV}$ were not from the $\mathrm{SiO}_{2}$ matrix nor from the Ge ncs. Instead, they came from the interface between the Ge ncs and the $\mathrm{SiO}_{2}$ matrix. These preliminary results show the feasibility of using PECVD techniques to prepare different size Ge ncs and confirm the existence of strain in Ge ncs. 


\section{Acknowledgments}

Professor Buwen Cheng from the Institute of Semiconductors, in the Chinese Academy of Science is acknowledged for his help with the TEM specimen preparations and Chris Boothroyd from the Center for Electron Nanoscopy, the Technical University of Denmark is appreciated for TEM examinations. The Danish Research Council of Technology and Production and the Danish Council of Strategic Research (\#09-072118/ENRG) are acknowledged for their financial support. The Danish Agency for Science, Technology and Innovation provided a grant (\#09-065038/FTP) for C. L. and for the UV-Raman instrumentation.

\#148333 - \$15.00 USD Received 27 May 2011; revised 11 Jul 2011; accepted 11 Jul 2011; published 18 Jul 2011

(C) 2011 OSA

1 August 2011 / Vol. 1, No. 4 / OPTICAL MATERIALS EXPRESS 651 